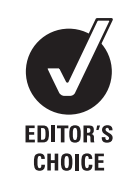

See Editorial, p 80

- Additional information is published online only at http:// sti.bmi.com/content/vol85/ issue2

${ }^{1}$ Indiana University School of Medicine, Indianapolis, Indiana, USA; ${ }^{2}$ Academic Edge, Inc, Bloomington, Indiana, USA

Correspondence to:

Mr R Goldsworthy, Research and Development, Academic Edge, Inc, 108 E 14th Street, Bloomington, IN 47408, USA; rick@academicedge.com

Accepted 16 November 2008 Published online first 22 December 2008

\title{
Formative design and evaluation of patient-delivered partner therapy informational materials and packaging
}

\author{
K McBride, ${ }^{1}$ R C Goldsworthy, ${ }^{2}$ J D Fortenberry ${ }^{1}$
}

\begin{abstract}
Objective: To develop and evaluate instructional and packaging materials for patient-delivered partner therapy (PDPT).
\end{abstract}

Methods: 64 patients participated from an urban US sexually transmitted infection (STI) clinic. The research comprised three phases: individual interviews to elicit attitudes and beliefs regarding PDPT and to assess the understanding of key STI-related concepts and terminology; the development and rapid validation of prototype instructional and packaging materials for PDPT and interviews to assess the effectiveness, acceptability and usability of the prototype materials. Thematic qualitative data analysis was used to examine interview responses. Results: Participants were willing to deliver and receive PDPT and several potentially important related beliefs were identified. Participants indicated substantial unfamiliarity with words associated with STI treatment and some variability in definitions of sex partners. PDPT informational materials differentially affected participant willingness to receive (positively) and deliver (negatively) PDPT, positively influenced self-efficacy and understanding and were perceived as easy to use.

Discussion: PDPT creates complex challenges for education, motivation and communication. Issues such as appropriate vocabulary and interpersonal trust may be amplified when responsibility for a medical proceduredispensation of treatment-is shifted to patients. STI PDPT implementation can be augmented with effective, high-quality informational and packaging materials; however, several challenges exist.

Untreated partners contribute to the high rates of re-infection among individuals with curable sexually transmitted infections (STI). ${ }^{1}$ However, studies of partner notification services found that as few as $20 \%$ of cases were actually interviewed by a public health worker. ${ }^{2}$ A potential solution to reducing re-infections and additional transmissions is for index patients to deliver antibiotics to their partners. Such patient-delivered partner therapy (PDPT) shifts services usually provided directly by a healthcare professional to patients themselves (although participants in PDPT are encouraged to be medically evaluated). ${ }^{3-5}$

PDPT appears to be a common but informal practice and is illegal or legally ambiguous in some areas. ${ }^{6-9}$ Guidelines for PDPT implementation exist and it is presently recommended for uncomplicated gonorrhea and chlamydia infections with populations other than men who have sex with men. ${ }^{17}$ Three randomised controlled trials and two observational studies have found various forms of PDPT to be effective for improving partner treatment for gonorrhea and chlamydia, although not all demonstrated statistically significant reductions in reinfection rates. ${ }^{10-14}$

Widespread, effective implementation of PDPT is contingent upon patient and partner willingness to participate: patients must deliver the medicine and partners must take it. A recent survey of the general population in the USA reported that healthcare consumers appear quite willing to participate as both deliverers and receivers of PDPT, with the majority of participants indicating a strong willingness to participate. ${ }^{3}$ It seems reasonable to suggest that perceptions of packaging and instructions accompanying PDPT medicine may affect willingness to participate as either a receiver or a deliverer, and Tun and colleagues ${ }^{15}$ provide some support for this possibility; however, no research exists that examines the design and evaluation of informational and packaging materials that accompany PDPT medication or prescriptions.

To address this gap, an established, user-centered, iterative development methodology ${ }^{16}{ }^{17}$ was used in combination with qualitative research conducted in a clinic setting to develop and evaluate prototype information and packaging materials to support PDPT delivery. This research was conducted in three phases. In the first phase, patient and partner needs were determined by qualitatively examining attitudes and beliefs regarding PDPT as well as an understanding of STI-related concepts and terminology. In the second phase, materials and packaging were formatively developed. Finally, in the third phase, the materials were evaluated in a clinic setting.

\section{METHODS}

\section{Participants}

Participants were patients aged 17-40 years attending an urban STI clinic in Indianapolis, Indiana, USA. Sixteen men and 16 women participated in phase 1 ; three men and two women participated in phase 2 and 13 men and 14 women participated in phase 3 . Forty-four per cent of the overall sample were Spanish-speaking Latino/a men and women $(n=28)$, whereas the remainder were English-speaking African American $(n=18)$ or white $(\mathrm{n}=18)$.

\section{Procedure}

Patients were recruited from the clinic waiting room by a researcher and taken to a private room to complete a 20-30 minute individual interview. 


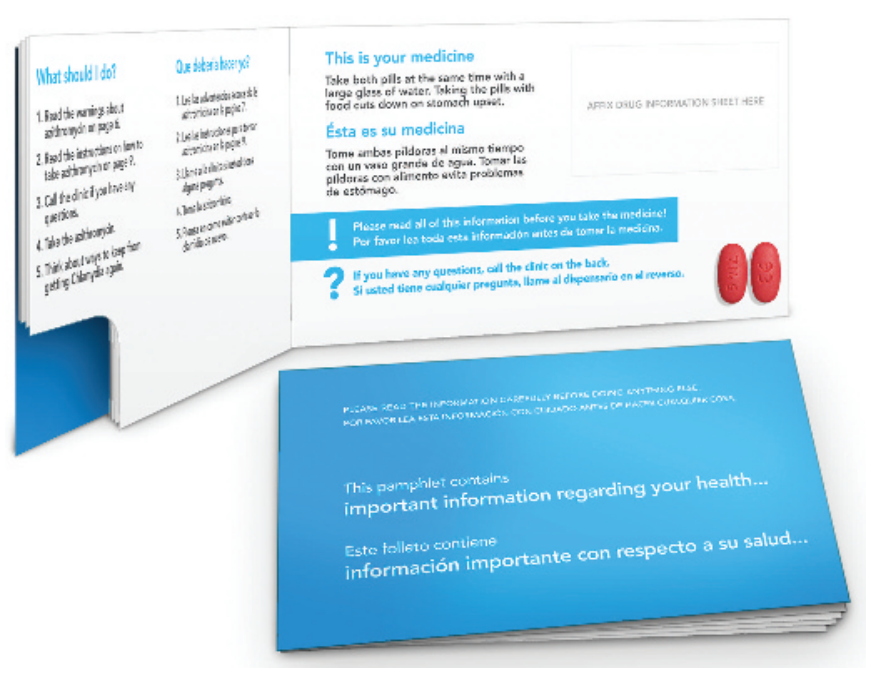

Figure 1 Images of mock patient-delivered partner therapy packaging.

Interviews were conducted in the participant's primary language (English or Spanish), were digitally recorded and were later transcribed for analysis. Participants were enrolled until thematic saturation was reached; that is, no new themes were emerging from the data. Participants received US $\$ 20$ for their time. The study was approved by the Institutional Review Board at Indiana University/Purdue University at Indianapolis.

\section{Measures}

Semistructured interview guides were designed for the study. In phase 1, participants responded to two counterbalanced hypothetical situations: delivering prescription medication intended to treat chlamydia to a partner and receiving such medication from a partner. Questions probed willingness, attitudes and beliefs regarding PDPT as well as understanding of words and concepts frequently encountered during STI PDPT implementation.

In phase 2, the researchers drew upon the phase 1 results, published guidelines, existing medication instruction and warning information and subject matter expert input to generate prototype STI PDPT informational materials and packaging. Development goals included low literacy accessibility (6th grade or less) in both English and Spanish, culturally appropriate phrasing, inconspicuousness and ease of use. The resulting medication package included background information regarding PDPT, disease information, medication usage instructions, advice to seek further testing and sources for additional information. A prototype was evaluated by additional clinic volunteers for acceptability, readability and comprehension, and was then modified for use in the phase 3 evaluation.

In phase 3 , participants followed a similar protocol to phase 1 , with the key difference being that when presented with the hypothetical situations, they were also given the PDPT information and packaging prototype. As in phase 1, willingness, attitudes, beliefs and understanding were assessed. In addition, questions specific to the impact, usability and understanding of the materials were asked.

\section{Analyses}

Spanish interviews were translated to English for analysis by a native Spanish speaker who conducted the interviews. In both phases 1 and 3, data were analysed to identify themes associated with the intention to deliver and receive PDPT medication. In
Table 1 Description of patient-delivered partner therapy packaging content, by page

\begin{tabular}{ll}
\hline Page no & Content \\
\hline 1 & $\begin{array}{l}\text { Cover: "This pamphlet contains important information regarding your } \\
\text { health..." (English and Spanish) } \\
\text { Introductory information and a warning to read all information before } \\
\text { taking medicine (English and Spanish) }\end{array}$ \\
3 & What's this all about? You may have chlamydia and this medicine can \\
4 & cure you (English and Spanish) \\
5 & What's chlamydia? (English and Spanish) \\
6 & What else should I know? (English and Spanish) \\
7 & Frequently asked questions (English) \\
8 & Frequently asked questions (Spanish) \\
9 & Warnings (English) \\
10 & Warnings (Spanish) \\
11 & What should I do? (English and Spanish) \\
12 & This is your medicine... (English and Spanish) \\
& "Be safe, get tested, get treated. Make a difference." Contact \\
\hline
\end{tabular}

the first stage of this analysis, open coding identified broad thematic categories within the participant responses. Subsequently, the coding scheme was revised to identify and organise subcategories, each representing specific themes within the broader categories. Finally, each interview was annotated with these resulting categories and subcategories. Summary statistics were generated for category counts as well as for phase 3 quantitative data from ratings and similar items.

\section{RESULTS}

\section{Phase 1}

Participants in phase 1 were largely willing to deliver medication to a sex partner(s) (87.5\%), but fewer participants were willing to receive medication from a sex partner (57\%). Benefits most frequently associated with both PDPT receipt and delivery were convenience and cost.

Lack of trust in a partner and the context of the relationship (eg, casual partner) were the primary reasons for being unwilling to receive medication from a partner. Participants also cited the need for testing and treatment by a healthcare provider before receiving treatment.

In relation to the willingness to deliver, many participants indicated that they would make an effort to deliver medication to sexual partners but that delivery would be contingent upon the ability to contact their partner(s). Participants also mentioned product safety as a major concern in terms of delivering the medicine, particularly the potential for side effects and allergic reactions, which is interesting because this theme did not arise in relation to receiving the medicine. Several participants indicated that they would not deliver PDPT because prescription medication should only be dispensed by a healthcare provider.

Participant understanding and application of commonly encountered sexual health-related concepts and terms was highly variable, especially definitions of "sex" and "sex partner". Many participants applied the term "sex partner", for example, only to a casual partner (ie, one night stand), whereas others used the term for a long-term partner only (ie, boyfriend/girlfriend), which suggests the need to clarify the terms during use. There was also a general lack of understanding of terms related to STI diagnosis and treatment such as "azithromycin" or "asymptomatic". Several participants did not understand "bacteria" and "infection". 
Table 2 Phase 3 interviews: perceptions of receiving and delivering patient-delivered partner therapy

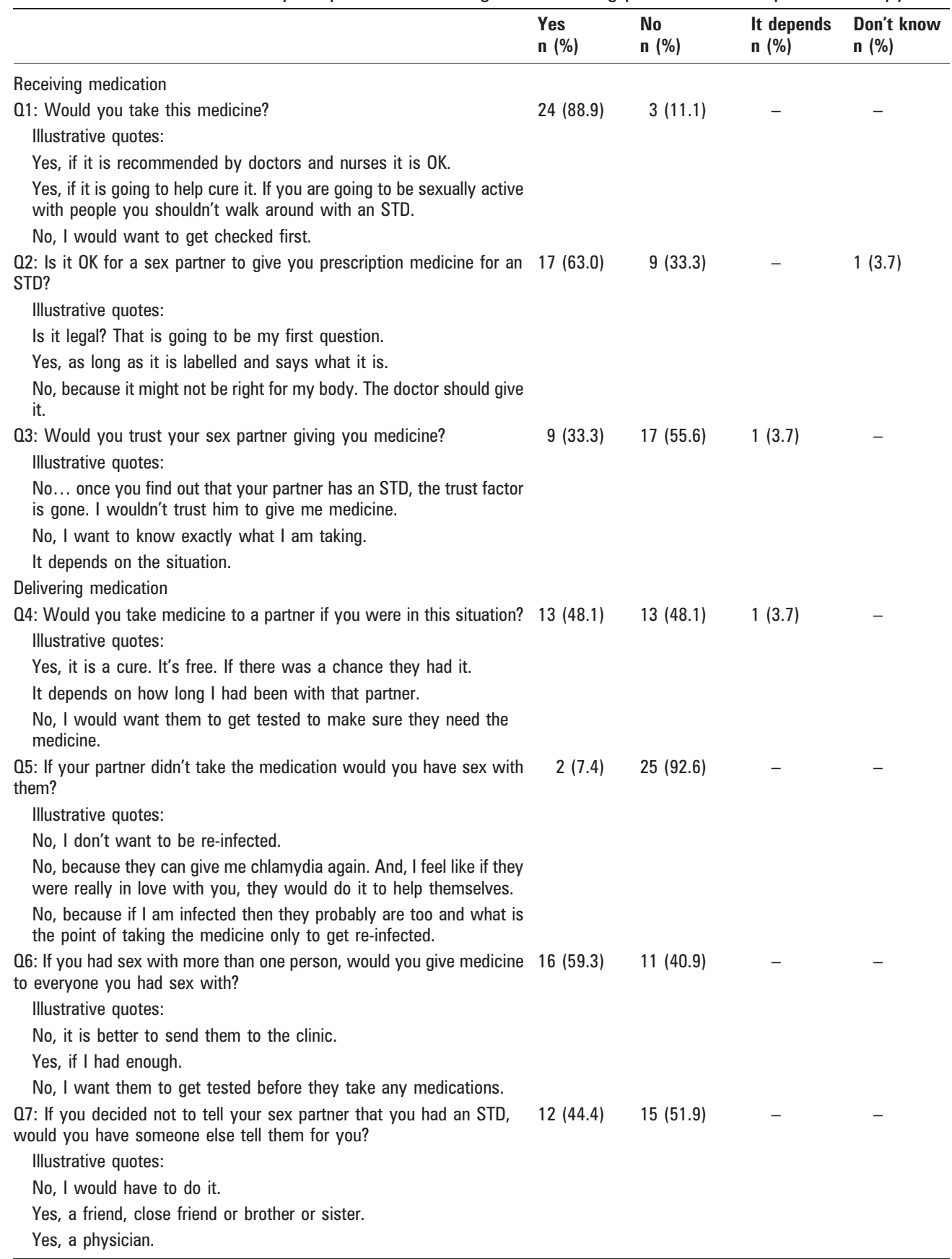

\section{Phase 2}

The prototype PDPT information and packaging is shown in fig 1 . The final package was a $2 \times 4$-inch stapled pamphlet containing six pages (12 printed sides). Medication was incorporated into the back page of the pamphlet, as a pushthrough-type blister pack. For the phase 3 evaluation efforts, the medication was represented by printed azithromycin tablets rather than the actual medication. Pamphlet content is delineated in table 1. More information regarding the development process and a portable document file (PDF) of the prototype are published as a supplement available online only.

\section{Phase 3}

Phase 3 repeated the phase 1 audience analysis by again exploring willingness, attitudes and beliefs regarding PDPT delivery and receipt, this time in the presence of the PDPT materials. Participants' perceptions of those materials were also assessed. Primary outcome measures included perceived ease of use, impact on PDPT performance and participants' understanding of the materials.

\section{Receiving}

Eighty-nine per cent of participants in phase 3 indicated that they would be willing to receive medication from a sex partner, with the primary reason for doing so being their own health. Among those who said they would decline the medicine, all cited the need to seek services from a healthcare provider as a primary reason.

Themes were similar to phase 1. More than half of participants indicated that they would not trust their sex 
Table 3 Willingness to participate in patient-delivered partner therapy, by phase

\begin{tabular}{lll}
\hline Action/phase & Phase 1 (\%) & Phase 3 (\%) \\
\hline Willingness to deliver & 87.5 & 48.1 \\
Willingness to receive & 57.0 & 88.9 \\
\hline
\end{tabular}

partner to give them medicine. The most common reason for lack of trust was the implication of infidelity associated with a STI diagnosis. Participants frequently stated that if a partner could not be trusted to be monogamous then medication received from that partner could not be trusted. Other participants noted that they would be less likely to trust a casual partner compared with a long-term partner. Apparent among both of these groups was the fear that partners might provide an illegal drug or unknown medication that could pose a danger to health.

Another reason for lack of trust was associated with perceptions of legitimacy. Individuals noted that their partner not being a healthcare provider meant that they were not viewed as a legitimate source of treatment. These participants viewed testing and treatment by a healthcare provider as the only legitimate form of care. However, two-thirds of participants believed that it was "OK" to receive medication from a sex partner. The remaining third cited the potential for adverse reactions (eg, drug allergies) and the need for testing and treatment by a healthcare provider as reasons for believing that PDPT was not "OK."

\section{Delivering}

Fewer than half of the respondents (48\%) said that they would be willing to deliver medication to a sex partner. Among these, the majority said that they would do so because they would want their partner(s) to be treated (see table 2). Participants also cited cost and convenience as factors influencing willingness to deliver. Of the participants who said they would not be willing to deliver the medication, the primary reason was the belief that the partner needed to be treated by a doctor. Both testing and the idea that medications should not be taken unless needed were primary themes among non-deliverers. Several themes not present in the first phase data also emerged: the legality of PDPT was questioned by some participants who noted that they would deliver medication only if it was legal.

Although stigma was not assessed directly in relation to the willingness to deliver medication, we did ask participants "what would be the hardest part about delivering the medicine to a partner?" The majority indicated that the stigma associated with having a STI and the association of STI with sexual nonmonogamy were significant barriers. Themes related to embarrassment and shame were common, as were themes related to relationship infidelity blame.

Because sex with untreated partners contributes to high reinfection rates, we assessed individuals' intent to have sex with an untreated partner. When asked if they would have sex with their partner again if the partner did not take the medication, $93 \%$ of participants responded "no", citing re-infection as the primary factor, which mirrored the phase 1 results.

In order to explore the significance of multiple sexual partnerships on the willingness to deliver medication, we asked participants if they would give medication to all their sex partners in the event that they had more than one partner. Sixty per cent of participants said they would take medication to each of their sex partners. The primary reason for doing so was the desire for that person to receive treatment. The majority of those who declined delivery did so because they believed that their partners needed to be seen by a healthcare provider.

Table 4 Phase 3 examples of qualitative information regarding patient-delivered partner therapy packaging

Question
01: Now that you have read the information, what do you think it means?
Illustrative quotes:
It is an explanation to someone who may or may not be sure they have the disease of what they should do and what they should
inform their partner of. (African-American man, 25)
Information about the medicine and how to prevent contracting chlamydia. (Hispanic man, 36)
It is trying to make people aware of the infection called chlamydia. How long you can have it, some of the symptoms and the
medicine that cures it. (African-American woman, 33)
02: What are the three most important things that this packaging is trying to tell you?
Illustrative quotes:
Get tested. There is a cure for it. Call the clinic. (Hispanic woman, 35)
Practice safe sex. Get tested for diseases. Safe way to take the medicine. (White man, 22)
Symptoms. How you can cure it. How it is spread. (African-American woman, 27)
03: What did you like MOST about the packaging?
Illustrative quotes:
It gives you the information that chlamydia doesn't always have symptoms. (African-American woman, 21)
Information about getting checked, that it is curable and the number to call. (White man, 40)
Easy to read. (White woman, 21)
04: What did you like LEAST about the packaging?
Illustrative quotes:
I have no clue what the names of the medication are. (White man, 40)
Not knowing how to pronounce the words. (African-American man, 21)
Nothing. It was pretty self-explanatory. (African-American woman, 20)
05: What would you CHANGE about the packaging?
Illustrative quotes:
More descriptive about symptoms. (African-American woman, 25)
How to pronounce the words. Maybe written in the margins or something. (African-American man, 21)
Nothing. Any information about a disease is good. (African-American woman, 32)


To understand better the potential resources for partner notification, we asked participants who they would ask to notify a sexual partner about a potential STI. Although $44 \%$ of the sample said "no-one", many of those responses were preceded by a comment that the participant would do it her/ himself. Of those who said they would have another person do it for them, the three most frequent resources for notification were family members, friends and healthcare providers.

\section{Packaging materials}

The participants in both phases 1 and 3 were asked about their willingness to deliver and receive PDPT. The primary difference between those phases was the presence of the materials in phase 3. Willingness to deliver was substantially lower in phase 3 than in phase 1 , whereas willingness to receive was substantially higher (see table 3 ). Moreover, $90 \%$ of participants agreed that the packaging materials would make them more able to approach partners than if they had only a prescription or medicine by itself.

In addition, $89 \%$ of participants agreed or strongly agreed that the materials were easy to understand. To assess participants' understanding of the packaging information and perceptions of usability further, five open-ended items (see table 4) were used. Participants largely interpreted the materials to mean that they may have been exposed to chlamydia, a curable disease, and that the medication provided with the materials was used to treat chlamydia infections. Specific themes centered around descriptions of chlamydia and its symptoms, medication side effects and contraindications, the importance of testing and/or treatment and resources for additional information. Participants also mentioned general messages such as "practice safe sex" and "get tested regularly for STI".

Aspects of the materials that participants liked most were also assessed. Most respondents indicated that they liked the information provided, frequently mentioning the details about chlamydia infection and the medication to treat infection. A few requested additional information about side effects and medication pronunciation.

\section{DISCUSSION}

Several issues of relevance to STI prevention and treatment efforts emerged. First, language and understanding is of concern. Some commonly used phrases such as "sexual partner" were insufficient in that some participants did not identify casual sexual contacts as "partners". This issue intersected with variation in definitions of "sex" as well as decreased motivation to deliver medication to or receive medication from a casual partner. This suggests that PDPT may be more effective for the prevention of re-infections within more established relationships than for the prevention of additional transmission within

\section{Key messages}

- Healthcare consumers are willing to participate in PDPT.

- Willingness appears to be related to partner trust, perceptions of medication legitimacy and concerns for product safety.

- Partner informational materials positively affected willingness to receive PDPT but negatively affected willingness to deliver PDPT.

- Further research regarding patient, partner and perhaps provider informational and educational materials is merited if PDPT practice is to become more widespread. sexual networks. Attention should be paid to increasing patient and partner understanding through provider counselling and/or PDPT materials.

Second, clinic visitors appear willing to engage in PDPT and several potentially important beliefs were identified, including partner trust, medication legitimacy and the perceived need to see a provider before treatment. Stigma, in particular, may be an important barrier to PDPT, especially on the part of the medication deliverer. Stigma in relation to STI care-seeking and treatment is well described but has received little attention in the emerging literature related to PDPT. This issue requires attention by providers in order to maximise the likelihood that the provided STI treatment will be delivered.

Third, packaging and instructions appear to be important. There is presently no accepted standard for such materials and we are unaware of any commercially available packaging or informational materials designed especially for PDPT. Clinical trials and anecdotal reports of typical PDPT practice implement an array of solutions ranging from simply handing over prescriptions and/or medications, to using plastic or paper bags accompanied by typed materials, to more formal accompanying materials such as letters on clinic stationery. Previous studies suggest that a certain level of "home-made" packaging is suboptimal, reducing the likelihood of patient and partner participation. ${ }^{15}$

In the present study, carefully designed informational materials affected the willingness to receive and, inversely, deliver PDPT as well as the themes identified in relation to such willingness. Willingness to receive may go up in the presence of commercial materials because perceptions of illegitimacy of the medication and other issues mentioned in the phase 1 data as reasons for not taking the medicine are, in fact, addressed and overcome in the presence of carefully designed packaging. On the other hand, having actual medicine in hand may deter willingness to deliver the medicine because the situation becomes more "real" and the warnings and instructions trigger patient reticence. Because patients were handed the PDPT packaging with little context and no counselling, we believe the latter finding is of less concern than the former, which strongly indicates that well-perceived materials may improve PDPT uptake among partners. The majority of participants also indicated that such materials would make it easier to approach partner(s).

The study sought to explore several subjective aspects of PDPT and to develop and evaluate prototype PDPT materials formatively. The researchers did not expect or intend a representative sample. The data are intended to portray issues that must be addressed in the successful implementation of PDPT and to provide guidance for the future development and use of PDPT informational and educational materials. Feasibility and dissemination research is not addressed here. The materials used were designed to be information plus medication packaging in one solution. This approach has merits but faces significant, although not insurmountable, manufacturing and regulatory hurdles; alternatively, the information components could be presented as standardised trifold brochures, a move that sidesteps the manufacturing issue but leaves packaging to individual practices. Further research is required to determine the most optimal and adoptable solutions.

\section{CONCLUSION}

PDPT is an acceptable and effective alternative clinical practice for partner treatment, the implementation of which is 
contingent on complex issues surrounding patient and partner willingness, attitudes and beliefs. Carefully developed informational materials appear to foster PDPT participation, although their relationship with willingness appears complex. Appropriate investment in research-based provider training and patient support, as well as the development of standardised packaging and/or information materials is necessary if PDPT is to be maximally effective. Well-designed, professional-appearing PDPT materials will improve participation. However, given the complex interpersonal issues involved in patient delivery and partner receipt, such improvements may be best realised as part of a broader system of adoption and implementation strategies and tools, addressing the needs of patients, partners and providers alike.

Acknowledgements: The authors would like to thank Arturo J Carrillo for conducting and transcribing the Spanish-language interviews.

Funding: This study was supported in part by contract no 200-2006-M-18977 from the Centers for Disease Control and Prevention (CDC). The information and opinions expressed herein do not necessarily reflect those of the CDC.

Competing interests: None.

Ethics approval: The study was approved by the Institutional Review Board at Indiana University/Purdue University at Indianapolis.

Contributors: $\mathrm{KMcB}$ participated in protocol design and conducted the qualitative interviews and, along with JDF, the analysis. RCG and JDF jointly conceived of the research efforts and guided protocol development. RCG is the principal investigator and has directed the design and evaluation of the materials.

\section{REFERENCES}

1. Centers for Disease Control and Prevention, National Center for HIV STD and TB Prevention. Expedited partner therapy in the management of sexually transmitted diseases: review and guidance, 2006. http://www.cdc.gov/std/treatment/ EPTFinalReport2006.pdf (accessed 10 Feb 2008).

2. Golden MR, Hogben M, Handsfield HH, et al. Partner notification for HIV and STD in the United States: low coverage for gonorrhea, chlamydial infection, and HIV. Sex Transm Dis 2003;30:490-6.
3. Goldsworthy RC, Fortenberry JD. Patterns and determinants of patient-delivered partner therapy uptake among healthcare consumers. Sex Transm Dis 2009;36:25-32

4. White PJ, Golden MR, Turner KM, et al. Can patient delivered partner therapy help us regain control of sexually transmitted infections in the UK? Sex Transm Infect 2006;82(Suppl 2):A2.

5. Golden MR. Expedited partner therapy for sexually transmitted diseases. Clin Infect Dis 2005;1:630-3.

6. Golden MR, Anukam U, Williams DH, et al. The legal status of patient-delivered partner therapy for sexually transmitted infections in the United States: a national survey of state medical and pharmacy boards. Sex Transm Dis 2005;32:112-14.

7. Hodge JG Jr, Pulver A, Hogben $\mathbf{M}$, et al. Expedited partner therapy for sexually transmitted diseases: assessing the legal environment. Am J Public Health 2008:98:238-43

8. Hogben M, McCree DH, Golden MR. Patient-delivered partner therapy for sexually transmitted diseases as practiced by U.S. physicians. Sex Transm Dis 2005;32:101-5.

9. Rogers ME, Opdyke KM, Blank S, et al. Patient-delivered partner treatment and other partner management strategies for sexually transmitted diseases used by New York City healthcare providers. Sex Transm Dis 2007;34:88-92.

10. Ramstedt K, Forrsman L, Johannisson G. Contact tracing in the control of genital Chlamydia trachomatis infection. Int J STD AIDS 1991:2:116-18.

11. Kissinger $\mathbf{P}$, Brown $R$, Reed $K$, et al. Effectiveness of patient delivered partner medication for preventing recurrent Chlamydia trachomatis. Sex Transm Infect 1998:74:331-3.

12. Golden MR, Whittington WL, Handsfield HH, et al. Partner management for gonococcal and chlamydial infection: expansion of public health services to the private sector and expedited sex partner treatment through a partnership with commercial pharmacies. Sex Transm Dis 2001;28:658-65.

13. Golden MR, Whittington WLH, Handsfield HH, et al. Effect of expedited treatment of sex partners on recurrent or persistent gonorrhea or chlamydial infection. N Eng/ J Med 2005:352:676-85

14. Kissinger $\mathbf{P}$, Mohammed $\mathrm{H}$, Richardson-Alston G, et al. Patient-delivered partner treatment for male urethritis: a randomized, controlled trial. Clin Infect Dis 2005:41:623-9.

15. Tun W, Walsh C, Siller J, et al. Acceptance of patient-delivered partner-therapy for syphilis among men who have sex with men (MSM). Proceedings of the 2004 National STD Prevention Conference; 8-11 March 2004, Philadelphia. Atlanta: Centers for Disease Control and Prevention, 2005.

16. Goldsworthy RC, Kaplan B. Warning symbol development: a case study on teratogen symbol design and evaluation. In: Wogalter MS, ed. Handbook of warnings. Mahwah, NJ: Lawrence Erlbaum, 2006:739-54.

17. Goldsworthy RC, Schwartz NC. Preliminary development and evaluation of a multimedia enhanced HIVSTD curriculum for middle schools. J Educat Multimedia Hypermedia 2008;17:413-44. 